\title{
Single Point Gradiometer for Planetary Applications
}

\author{
José Luis Mesa , Marina Díaz Michelena, David Ciudad , Whitney Schoenthal \\ Michael E. McHenry, Marco Maicas , and Claudio Aroca
}

\begin{abstract}
We have designed and fabricated a microelectromechanical device, based on the alternating field gradient concept, to measure surface magnetic field gradient on planets. Its sensitivity is $4 \times 10^{-4} \mathrm{~T} / \mathrm{m}$, which is appropriate for magnetite outcrops and areas with rocks formed at different stages recording geomagnetic field reversals. We present the results obtained with three different prototypes.
\end{abstract}

Index Terms-Magnetic instruments, gradient methods, microelectromechanical system, microsensors.

\section{INTRODUCTION}

Crustal sources are an important contribution to planetary magnetic fields (Earth) and the primary contribution when no global field remains (Mars/Moon) [Langlais 2004]. To unambiguously distinguish these sources is a complex problem. Measurements at different altitudes or measuring derivatives of the field would improve the convergence of the solution.

This study complements current magnetic ground surveys with instruments capable of measuring the magnetic field gradient. The field produced by Earth lithospheric anomalies ranges from -3700 to $8300 \mathrm{nT}$ at a low Earth orbit of $450 \mathrm{~km}$ from the surface. On ground, high (spatial) resolution surveys in geological structures with an associated magnetic signature (magnetite outcrops, volcanoes, impact craters, basaltic intrusions, etc.) range from 0.1 to $100 \mu \mathrm{T}$ [Michelena 2015]. Resolutions on the order of $10^{-8}-10^{-6} \mathrm{~T} / \mathrm{m}$ are necessary for accurate on-ground geophysical surveys: to identify magnetic minerals like magnetite, pyrrhotite, and hematite, and different mineral phases (titanomagnetites) on Earth [Sanz 2011]. Rougher sensitivities are still interesting for strong field contrasts like the magnetite outcrop of El Laco, Chile (>100.000 nT in $<1 \mathrm{~m}$ ) [Michelena 2015], some areas with high remanence rocks formed during different ages, including geomagnetic field reversals, and probably for the strong magnetic anomalies reported on Mars [Dunlop 2005].

We describe a single point gradiometer named for the short spatial base of the measurements $(\mathrm{mm})$ compared with the wavelength of most magnetic anomalies in geological structures (cm to $\mathrm{km}$ ).

The magnetic induction vector $\vec{B}$ will be referred as the magnetic field. The magnetic field gradient is composed of nine elements represented by a symmetric tensor $\overrightarrow{\nabla B}$, where $\frac{d B_{i}}{d x_{j}}=\frac{d B_{j}}{d x_{i}}$, in the absence of electrical currents or time varying electric fields $(\nabla \times \vec{B}=0)$. Here, we focus on a nondiagonal component of the tensor. However, the system can be easily adapted to measure the other five components of the gradient.

\section{METHODOLOGY}

The determination of magnetic field gradient is based on ideas used in alternating gradient/force magnetometers (AGM/AFM). In an AFM, a sample is vibrated by means of a magnetic force and the vibration amplitude is measured by optical, capacitive, or other contactless detectors. Many micromagnetic devices [Moreland 2003] have been constructed based on principles of AGM, torsional oscillator [Yin 2013], or the symmetric vibrating-sample magnetometer (VSM) [Ziljstra 1967]. In the past, we have used AFMs [Michelena 2000] to measure magnetic properties of sputtered thin films of low magnetic moment due to the comparative advantages of AFM as compared with VSM: lower noise and better resolution.

Similar devices have been previously reported including a gradiometer developed by Sunderland [2008] based on an aluminum wire ( $0.25 \mathrm{~m}$ length) with better resolution which has been qualified for space applications.

Three AFM-based microelectromechanical (MEMS) prototypes of low noise and high resolution have been developed to measure the gradient of the magnetic field [Ciudad 2009]. The physical principle and characteristics of the device are described in the following. The potential energy $U$ of a magnetic dipole moment $\vec{m}$ in the presence of a magnetic field is

$$
U=-\vec{m} \cdot \vec{B} .
$$

The magnetic force $\vec{F}$ is derived as the gradient of the potential energy:

$$
\vec{F}=-\nabla U=\nabla(\vec{m} \cdot \vec{B}) .
$$

Assuming that a magnetic dipole

$$
\vec{m}=m_{x} \vec{i}
$$

is attached to a mechanical cantilever, in the presence of a magnetic field with a single component in the $x$ direction

$$
\vec{B}=B_{x} \vec{i}
$$

which varies spatially along the $z$ direction

$$
\overrightarrow{\nabla B}=\frac{\partial B_{x}}{\partial z} \vec{k}
$$




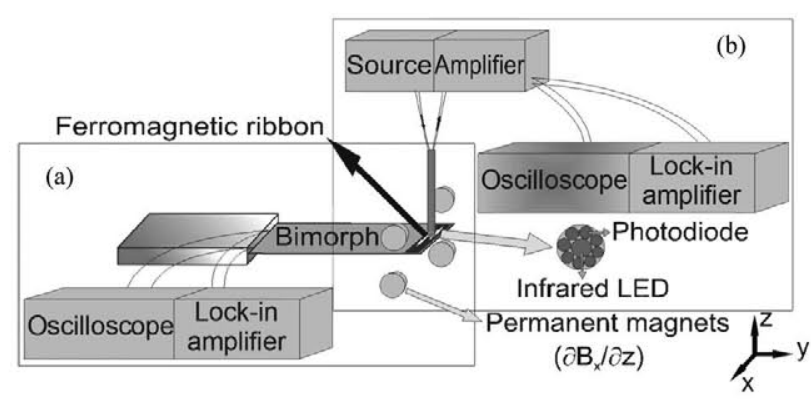

Fig. 1. Diagram for setups: (a) piezoelectric detection method (prototype 1); (b) optoelectronic detection (prototypes 2 and 3).

Table 1. Ferromagnetic ribbons properties.

\begin{tabular}{lcc}
\hline \hline & FMA $\backslash 2826 \mathrm{MB}$ & $\mathrm{FMB} \backslash 2705 \mathrm{M}$ \\
\hline Thickness & $29 \mu \mathrm{m}$ & $22 \mu \mathrm{m}$ \\
Length & $20 \mathrm{~mm}$ & $20 \mathrm{~mm}$ \\
Width & $1.5 \mathrm{~mm}$ & $1.5 \mathrm{~mm}$ \\
Saturation Induction & $0.88 \mathrm{~T}$ & $0.77 \mathrm{~T}$ \\
Magnetostriction & $12 \mathrm{ppm}$ & $<0.5 \mathrm{ppm}$ \\
\hline \hline
\end{tabular}

the force contribution is (only $z$ direction)

$$
F_{z}=m_{x} \frac{\partial B_{x}}{\partial z} .
$$

In this setup (see Fig. 1), commercial soft magnetic materials by Metglas (see Table 1) are attached at the end of a cantilever. The magnetic material is magnetized in an alternating field $B_{x}(t)$, acquiring an alternating dipole $m_{x}(t)$.

In a magnetic field gradient [see (5)], the free end of a cantilever experiences an alternating force along $z$-axis [see (6)] and vibrates. If the frequency of the force is tuned at the cantilever's mechanical resonance frequency, in the absence of an external gradient, the amplitude of the vibration is maximized and the phase exhibits a maximum shift $\left(\theta=180^{\circ}\right)$. In an external field gradient parallel to the exciting gradient [see (5)], the cantilever oscillation will change in amplitude and phase.

Thus, knowing the exciting gradient and magnetic moment amplitude of the sample, it is possible to determine external gradients, by monitoring the vibrational properties.

In our prototypes, the vibration is characterized by piezoelectric effect and optically. The cantilever is a bimorph, which consists of two stacked piezoelectric plates of lead zirconate titanate (PZT): of dimensions $60 \times 20 \times 0.7 \mathrm{~mm}^{3}$-cantilever $\mathrm{A}$; and $40 \times 20 \times 0.7 \mathrm{~mm}^{3}$-cantilever B, inversely polarized to bend the system. An estimation of the force resolution limit for a vibrating cantilever due to thermal noise is given by Stowe [1997]. Extrapolating the results of the magnetic force produced by the magnetization of the ribbons on the cantilever $\left(\approx 10^{-6} \mathrm{~N}\right)$ and comparing it with the limit force resolution $\left(\approx 10^{-10} \mathrm{~N}\right)$, we assume that we are far enough from this limit to ignore thermal noise effects in our results. The changes in vibration are read by the induced piezoelectric voltage.

The optical detection is based on the different light cone, i.e., light intensity received by a photodiode, after being reflected by a surface, depending on the relative distance between the reflecting surface and the optical collector [Lucas 2009]. This method is preferred to beam bounce [Yin 2013] and others for its compactness. The light source is a light emitting diode (LED) and the detector is a photodiode [see Fig. 1(b)]. The light is transmitted to the vibrating structure by an optical fiber and collected by six optical outer fibers (daisy configuration array) to guide it back to the photodetector. The array is placed perpendicular to the sample and the LED light reflects off the sample and is collected by the outer fibers. This system provides higher resolution $(4 \mathrm{~nm})$ than piezoelectric detection $(1 \mu \mathrm{m})$.

\section{EXPERIMENTAL RESULTS}

Based on the methodology described in Section II, three different macroscopic setups have been evaluated in terms of their magnetic properties: resolution, long term stability, and sensitivity, and other aspects as power consumption and complexity. The three devices have been characterized in the presence of an external field gradient produced by a magnetized sample approached at a controlled distances from the vibrating cantilever, previously quantified in the position of the cantilever by means of a magnetometer. The corresponding external field gradient is obtained by measuring the field at different distances. Along the approach direction, changes in amplitude and phase are monitored. The changes in the oscillation amplitude and phase are represented as a function of the field gradient created by the sample, demonstrating linear dependence and sign sensitivity. Next, we describe the three prototypes developed and their main characteristics.

\section{A. Prototype 1}

In this prototype, the mechanical structure is the Cantilever A made of a bimorph material. On its surface it has a set of six Metglas 2826 MB magnetic ribbons FMA (see Table 1), attached close to the free end of the cantilever such that the long dimension of the ribbons is set perpendicular to the length of the cantilever. The ribbons are magnetized along their length with an alternating magnetic field so that in each cycle, an alternating dipole is set in both directions of the $x$-axis in the free end of the cantilever. Metglas ribbons were selected as a commercial soft magnetic material of high permeability and low coercivity, which facilitates ac magnetization.

Due to the low resonance frequency of the system $(42 \mathrm{~Hz})$, an electromagnet was used to magnetize the ribbons $(10 \mathrm{mT}$ max.). A set of four permanent magnets were used to provide the magnetic field gradient [setup is depicted in Fig. 1(a)]. In this first prototype, the vibration is monitored by means of piezoelectric effect. If the bimorph is made of two piezoelectric layers, when the cantilever vibrates, the layers stretch and contract, setting an electrical voltage across them. The oscillating voltage is measured with a lock-in amplifier. This system was subjected to well-known external gradients in a range of $0.25 \mathrm{~T} / \mathrm{m}$. Fig. 2 shows the results in amplitude and phase.

This first prototype shows limitations in both excitation and detection. On the one hand, the dimensions of the soft magnetic ribbons make their magnetization to saturation difficult 


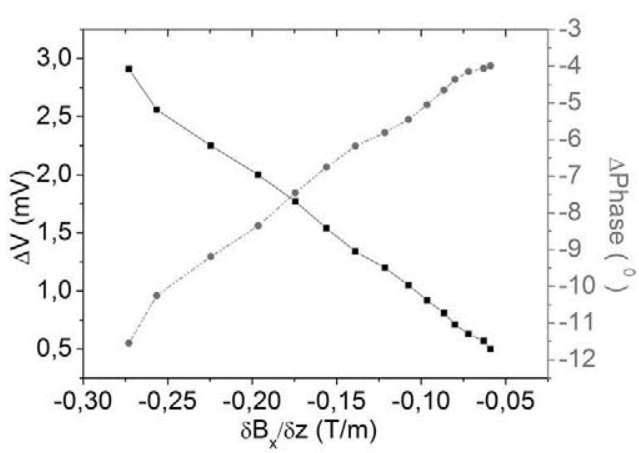

Fig. 2. Prototype 1 results: amplitude (black) and phase (grey).

due to the relatively high demagnetizing factor, and the power budget is excessive (electromagnet consumption up to $20 \mathrm{~W}$ ). Also, $2826 \mathrm{MB} / \mathrm{FMA}$ (see Table 1) ribbon is highly magnetostrictive (12 ppm) compared to other ribbons like $2705 \mathrm{M} / \mathrm{FMB}$ $(<0.5 \mathrm{ppm})$ and this may imply noncontrolled effects. On the other hand, the piezoelectric detection has a restricted sensitivity with resolutions on the order of 1-10 $\mu \mathrm{m}$.

The external gradient used in this prototype is negative, and adds to the permanent gradient (generated by the magnets), which is also negative, producing an increase of the signal amplitude with an increase of the external gradient amplitude (see Fig. 2). In case the permanent gradient is positive (prototypes 2 and 3), a negative value of the external gradient would generate a decrease in the oscillating amplitude, as it will be shown in the calibration curve for prototype 3 [see calibration inset in Fig. 4(a)]. This demonstrates the ability of the system to be sign sensitive to the voltage changes.

\section{B. Prototype 2}

This device improves some of the limitations of the former prototype. In this case, the ribbon used is FMB, Metglas $2705 \mathrm{M}$ (low magnetostriction), magnetized by means of an electrical current instead of coils to improve the power efficiency, and on the other hand, the detection is carried out optically to improve the resolution. The complexity of this setup is slightly higher than that of prototype 1 .

The cantilever is the same bimorph material (cantilever B) to homogenize the setups for a proper comparison, and the gradient is generated with magnets as in the previous prototype. Prior to attachment to the cantilever, the ribbon is annealed with current for $69 \mathrm{~min}$ at $780 \mathrm{~mA}$. After the annealing, the sample presents an induced helical magnetic anisotropy [Rodríguez 1998]. When an alternating current is set through the ribbon along its length, the ribbon acquires opposite and transversal magnetization (in the $y$ axis) on the two surfaces. The two magnetic domains are separated by a Bloch wall with a weak magnetization in the direction of the current $\left(m_{x}\right)$ making possible the desired oscillation [see Fig. 3(a)]. Note that in this case the force intensity is several orders of magnitude lower than in prototype 1 . This has a strong influence on the stability of the response, which is very close to the limit in resolution of the device in this case, since the small oscillations of the cantilever (minimum force estimated in $\approx 10^{-9} \mathrm{~N}$ ) are close to the ther-
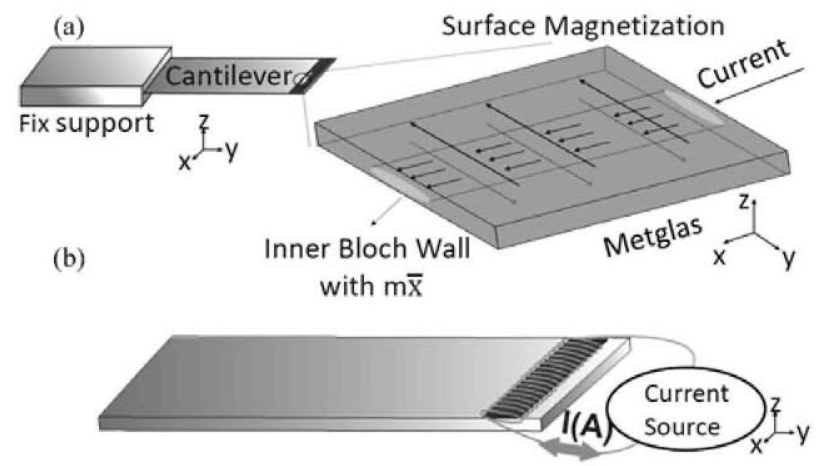

Fig. 3. (a) Illustration of the magnetization of a section of the annealed ribbon for a circulating current. (b) Schematic design for the wound ribbon of prototype 3 .

mal mechanical noise. The power consumption of the system is $20 \mathrm{~W}$.

The optical system consists of a bundle of optical fibers: one of $1 \mathrm{~mm}$ diameter and six of $0.5 \mathrm{~mm}$ diameter in a daisy configuration (see Section II). This system provides higher resolution $(4 \mathrm{~nm})$ than the piezoelectric detection $(1 \mu \mathrm{m})$.

The changes in oscillation amplitude of the cantilever when a magnetic field gradient is generated by approximating a permanent magnet to the system follow a linear behavior as shown for prototype 1. Phase data present very low stability due to the low magnetic moment of the wall.

\section{Prototype 3}

The third prototype was designed to have a more robust configuration than the previous ones. It is intended to generate a more intense and stable magnetic moment, power efficiently, using a microsolenoid wound around the ribbon.

The $2705 \mathrm{M}$ ribbon (FMB) is driven to saturation by means of the circulation of an alternating current through the microsolenoid [see Fig. 3(b)]. Due to the higher volume of the ribbon compared to that of the Bloch wall of prototype 2, the magnetic moment is much higher. Also, the magnetic ribbon reaches saturation easier than in prototypes 1 and 2 with low power consumption $(0.5 \mathrm{~W})$. Cantilever A oscillation monitoring is performed by the optical method described in Section II.

Fig. 4 shows the results for this prototype. In this case, the field gradient is generated by moving a piece of magnetite toward the system. The inset shows the response in amplitude and phase of this prototype in the presence of a gradient generated by a magnet like in the previous cases. The graphs show a more stable response with better sensitivity than the former ones. The response is linear. The deviations from linearity are attributed to a nonhomogeneous gradient along the long ribbon.

Note in Fig. 4(a) that the external gradient is negative for the calibration curve and positive for the magnetite curve. When $\frac{\partial B_{x}}{\partial z}=0$, the signal for the phase must be $\theta=180^{\circ}$. The intercept values of the phase are $\theta=172^{\circ}$ for the calibration curve and $\theta=171^{\circ}$ for the magnetite curve, and the slopes show different sign for negative and positive external gradients. This result shows sign dependency of the phase with the external magnetic field gradient, proving sign sensitivity of the phase. 


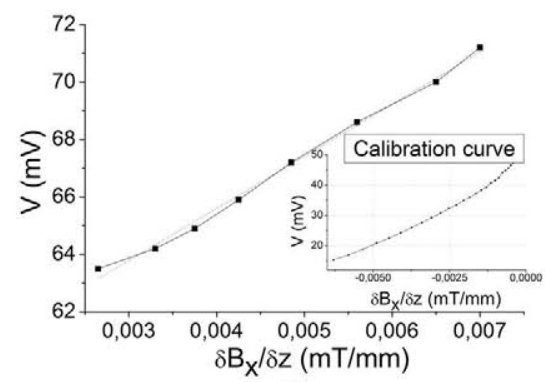

(a)

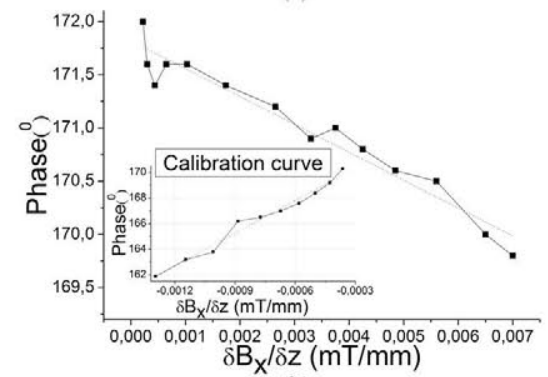

(b)

Fig. 4. Amplitude (a) and phase (b) changes of prototype 3. Main and embedded calibration curves share axis' labels.

Table 2. Prototypes sensitivity.

\begin{tabular}{lcccc}
\hline \hline Prototype & $\begin{array}{c}\text { Amp.Sens. } \\
\mathrm{mV} /(\mathrm{T} / \mathrm{m})\end{array}$ & $\begin{array}{c}\text { Lin. error } \\
\mathrm{mV} /(\mathrm{T} / \mathrm{m})\end{array}$ & $\begin{array}{c}\text { Ph. sens } \\
\% /(\mathrm{T} / \mathrm{m})\end{array}$ & $\begin{array}{c}\text { Lin. Error } \\
\% /(\mathrm{T} / \mathrm{m})\end{array}$ \\
\hline 1 & 10.8 & 0.8 & 33.8 & 0.9 \\
2 & 12.4 & 0.3 & $\sim$ & $\sim$ \\
3 & $1.32 \cdot 10^{3}$ & 80 & 262 & 20 \\
\hline \hline
\end{tabular}

\section{DISCUSSION}

Table 2 shows the sensitivity and its linearity error in amplitude and phase of the different prototypes, i.e., the slope of the linear fit of the responses to the field gradient.

The resolution (minimum detectable gradients) of the prototypes are: $0.05,0.03$, and $4 \times 10^{-4} \mathrm{~T} / \mathrm{m}$ for prototypes 1,2 , and 3 , respectively. These estimations were measured with resolution of $2-10 \%$ (full scale). Resolution of prototype 3 is measured with a function analyzer (noise spectrum).

In the following, a comparison is made between the features of the different prototypes in terms of resolution, stability, and complexity. It can be seen from Table 2 and Fig. 2 that prototype 1 presents good behavior with respect to the external magnetic field gradient, with a maximum resolution of $0.05 \mathrm{~T} / \mathrm{m}$. The resolution however, is still three to four orders of magnitude away from the objective, which is around $10^{-6}-10^{-5} \mathrm{~T} / \mathrm{m}$.

The limitations of this setup are due to two main factors. 1) The high demagnetization factor of the ribbons (limited by the width of the bimorph $-20 \mathrm{~mm}$ ), which makes the magnetization to saturation difficult. 2) The low piezoelectric constant (around $7 \times 10^{-10} \mathrm{~m} / \mathrm{V}$ according to the manufacturer), which sets a low voltage in the bimorph.

Prototype 2 shows higher stability than prototype 1 . The major improvement is the change in the detection method: from piezoelectric to optoelectronic. Furthermore, the possibility to replace the ceramic of the cantilever by a material with a more suitable shape and a lower Young modulus (thick silicon cantilever) makes it very interesting.

The resolution of $0.03 \mathrm{~T} / \mathrm{m}$ reached by this prototype is limited by the small magnetic moment in the desired direction achieved with this configuration. The moment is limited to the weak magnetization and the low volume of the inner Bloch wall. The result is considered a success in terms of the detection, and it motivates the use of optical.

Best results and features have been reached with prototype 3 due to its lower consumption and robustness, regarding the objective of this device. The length of the ribbon makes it easy to saturate via a microsolenoid. Resolution of $4 \times 10^{-4} \mathrm{~T} / \mathrm{m}$ involves a great improvement over the previous prototypes. This technique will be considered the starting point in future developments. Furthermore, the complexity of its fabrication is moderate and the power consumption is compatible with that of a rover payload $(1 \mathrm{~W})$.

\section{ACKNOWLEDGMENT}

The work of J. L. Mesa was supported by INTA training Grant TS09/12. This work has been done in the frame of PRI-PIBUS-2011-1150 and 1182 grants of the subprogram of international and bilateral projects of the Spanish Ministry of Science and Innovation. The work at CMU is supported by the National Science Foundation through Materials World Network under Grant DMR1106943.

\section{REFERENCES}

Ciudad D, Aroca C, Sánchez P, Prieto J L (2009), "Gradiometro de campo magnético, mecánico y miniaturizable, y su funcionamiento," ES 2327595 A1 Spanish Patent. [Online]: Available: http://bopiweb.com/elemento/20798/

Michelena M D, Kilian R (2015), "Magnetic signatures of the orogenic crust of the Patagonian Andes with implication for planetary exploration," Phys. Earth Planet, submitted for publication.

Michelena M D, Sánchez P, López E, Sánchez M C, Aroca C (2000), "Optical vibrating sample magnetometer" J. Magn. Magn. Mater., vol. 215-216, pp. 667-679, doi: 10.1016/S0304-8853(00)00256-0.

Dunlop D J, Arkani-Hamed J (2005), "Magnetic minerals in the Martian crust," J. Geophys. Res., vol. 110, E12S04, doi: 10.1029/2005JE002404.

Langlais B, Purucker M E, Mandea M (2004), "Crustal magnetic field on Mars," J. Geophys. Res., vol. 109, E02008, doi: 10.1029/2003JE002048.

Lucas I, Michelena M D, del Real R P, de Manuel V, Plaza J A, Duch M, Esteve J, Guerrero H (2009), "A new single-sensor magnetic field gradiometer," Sens. Lett., vol. 7, pp. 563-570, doi: 10.1166/sl.2009.1110.

Moreland J (2003), "Micromechanical instruments for ferromagnetic measurements," J. Phys. D, Appl. Phys., vol. 36, pp. R39-R51, doi: 10.1088/0022-3727/36/5/201

Rodríguez M, López E, Sánchez M C, Aroca C, Sánchez P (1998), "Irreversibility and magnetization dynamics in samples with helical anisotropy," J. Appl. Phys., vol. 83, pp. 4835-4837, doi: 10.1063/1.367279.

Sanz R, Cerdán M F, Wise A, McHenry M E, Díaz-Michelena M (2011), "Temperature dependent magnetization and remanent magnetization in pseudo-binary $\mathrm{x}\left(\mathrm{Fe}_{2} \mathrm{TiO}_{4}\right)-(1-\mathrm{x})\left(\mathrm{Fe}_{3} \mathrm{O}_{4}\right)(0.30<\mathrm{x}<1.00)$ titanomagnetites," IEEE Trans. Magn., vol. 47, pp. 4128-4131, doi: 10.1109/TMAG.2011.2157903.

Stowe T D, Yasumura K, Kenny T W, Botkin D, Wago K, Rugar D (1997), "Attonewton force detection using ultrathin silicon cantilevers," Appl. Phys. Lett., vol. 71, pp. 288-290, doi: 10.1063/1.119522.

Sunderland A, Veryaskin A V, McRae W, Ju L, Blair D G (2008), "Direct string magnetic gradiometer for space applications," Sens. Actuators A, Phys., vol. 147, pp. 529-535, doi: 10.1016/j.sna.2008.06.014.

Yin X, Jiao Q, Lu Y, Liou S-H (2013), "MEMS torsion oscillator magnetic field sensor," IEEE Trans. Magn., vol. 49, pp. 3890-3892, doi: 10.1109/TMAG.2013.2252153.

Ziljstra H (1967), Experimental Methods in Magnetism. Amsterdam, The Netherlands: North-Holland. 